\title{
KUNO-Kids birth cohort study: rationale, design, and cohort description
}

\author{
Susanne Brandstetter ${ }^{1 *}$ (D), Antoaneta A. Toncheva', Jakob Niggel', Christine Wolff', Silvia Gran', \\ Birgit Seelbach-Göbel ${ }^{2}$, Christian Apfelbacher ${ }^{3}$, Michael Melter ${ }^{1}$, Michael Kabesch ${ }^{1}$ and the KUNO-Kids study group
}

\begin{abstract}
Background: Birth cohort studies can contribute substantially to the understanding of health and disease - in childhood and over the life course. The KUNO-Kids birth cohort study was established to investigate various aspects of child health, using novel omics technologies in a systems medicine approach.

Results: After 3 years of recruitment, 2515 infants and their families have joined the study. Parents with higher education are overrepresented as in many other birth cohorts and are more likely to complete follow-up assessments via self-report questionnaires. The vast majority of participants consented to clinical examinations of their child and to the non-invasive collection of diverse biosamples, which were processed specifically for their integrated use in omics technology covering genomics, epigenomics, transcriptomics, metabolomics, and microbiome analyses of the skin, oral cavity, and stool.

Conclusions: The data and diverse biomaterial collected in the KUNO-Kids birth cohort study will provide extensive opportunities for investigating child health and its determinants in a holistic approach. The combination of a broad range of research questions in one study will allow for a cost-effective use of biomaterial and omics results and for a comprehensive analysis of biological and social determinants of health and disease. Aiming for low attrition and ensuring participants' long-term commitment will be crucial to fully exploit the potential of the study.
\end{abstract}

Keywords: Birth cohort, Child health, Study design, Participation, Omics

\section{Background}

Birth cohort studies have contributed significantly to advancing medical knowledge about childhood and adult health. A particular strength of such studies is their potential to identify mechanisms of health and disease over the life course through prospective followup. Although bias and confounding are serious problems in observational research, associations between exposures and outcomes can be established [1], which might even provide evidence for causal pathways. Links between exposures from early childhood to subsequent disease outcomes in adulthood are of particular relevance [2]. The number of available birth cohorts is substantial (birthcohorts.net $[3,4,5]$ ), but findings cannot always be readily transferred from one population to another

\footnotetext{
* Correspondence: susanne.brandstetter@ukr.de

${ }^{1}$ University Children's Hospital Regensburg (KUNO-Clinics), University of Regensburg, Clinic St. Hedwig, Steinmetzstr. 1-3, 93049 Regensburg, Germany

Full list of author information is available at the end of the article
}

because environmental, socio-economic, and cultural factors differ greatly between populations. Due to rapid lifestyle, societal, and environmental changes in many societies over the past decades, birth cohorts can become outdated within just a few years, no longer being applicable to the present generation. To consider recent developments within the population and make health decisions on the personal and population level based on knowledge rather than on assumptions, the availability of current data gained through state of the art assessment is crucial.

Thus, in a contemporary birth cohort study, recent socio-cultural developments in Germany ought to be reflected, including the shifting roles of motherhood and fatherhood, facilitated by financial support for parental leave $[6,7]$, increasing availability of early child care [8], rising birth rates [9], and widespread digital technology in work and home environments [10, 11]. Furthermore, medical knowledge has progressed significantly in many fields, leading to new health promotion and prevention programs. Finally, novel scientific methods and research 
paradigms have emerged rapidly in recent years driven by a big leap in omics technologies allowing measurements of countless molecular parameters describing the genome, the epigenome, the transcriptome, and the metabolome as well as the microbiome of an individual with an unprecedented abundance of information. On the other hand, a vast amount of environmental influences (exposome) can now be measured with novel tools such as satellite data, personal tracking devices, and advanced biochemical screening tools [12]. Taken together, an integrated systems biology approach to better understand the development of health and disease has now become feasible for birth cohort studies at a level previously not possible.

Against this background, the KUNO-Kids birth cohort study was established with three major objectives: (a) to contribute to the understanding of current child health using novel omics technologies in a systems medicine approach, (b) to identify novel modifiable factors of child health and opportunities for prevention, and (c) to provide a platform for investigating the feasibility and effectiveness of targeted interventions. Here, we provide an overview of the rationale and design of the KUNOKids birth cohort study, describing the characteristics of study participants after 3 years of recruitment and the procedures for sampling and processing biomaterial for omics analysis, as first analyses based on the current dataset from this study will soon be available.

\section{Methods}

\section{Design}

KUNO-Kids is a multi-purpose birth cohort study, which aims at investigating a wide range of exposures and outcomes. Data are collected not only from the index child (or children in the case of multiples), but also from his/her family (including mother, father, and siblings). The inclusion in the study starts immediately after birth of the index child. The baseline assessment takes place during the short hospital stay of mother and child directly after birth. Regular follow-up assessments are conducted when the index child is 4 weeks old, 6 months old, and at each birthday. Figure 1 provides an overview of the study design and the time points of data collection.

\section{Catchment area, setting, and recruitment of study participants}

The study is based in Eastern Bavaria (Regensburg, Germany). The catchment area is characterized by the city of Regensburg (164,000 inhabitants, among them 32,000 university students) and its predominantly rural adjacent regions. Rates of unemployment are among the lowest in Germany [13], and the population size is currently rising [14]. The clinic St. Hedwig is a modern children's and women's hospital affiliated with Regensburg University where about two thirds of the children from the region are born (approximately 3000 births per year). Being a tertiary perinatal center, the proportion of high-risk births is large.

After childbirth, mothers at the clinic St. Hedwig are approached by trained study personnel and are informed about the study aims and procedures. Mothers are eligible for enrolment in the study if they are 18 years of age or older and if they are able to provide informed consent (i.e., basic German language skills for the comprehension of study procedures are required). There are no further exclusion criteria. Only one child (or twin pair or triplet) per family is included in the study with the index birth event which initiated recruitment. Characteristics of siblings are assessed by questionnaires in the course of the study. Reasons for non-participation are assessed in mothers who do not consent to

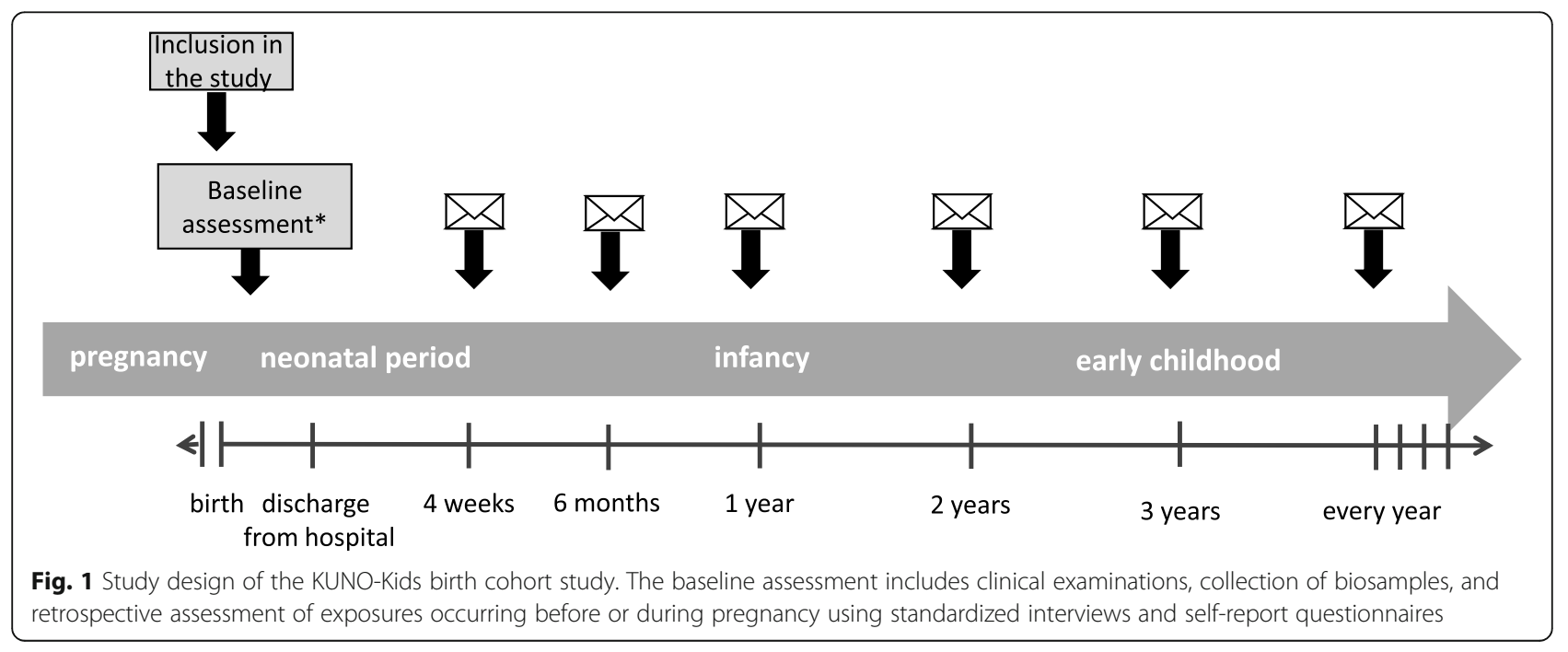


participate in the study. Recruitment began in June, 2015, and will be continued for at least five more years.

\section{Sample size and participant flow}

Within this timeframe, the study is designed to enroll at least 5000 and not more than 10,000 newborns and their families. Loss-to-follow-up was assumed to be substantial, particularly for the time period between inclusion at birth and the first follow-up. Therefore, a large number of study participants were considered necessary to provide adequate sample size for a majority of the proposed research questions. However, an a priori sample size estimation was not considered useful as the KUNO-Kids birth cohort is intended to cover a wide array of research questions which relate to different outcomes at various time points, thus leading to a large range of power estimates.

\section{Measurements and data collection}

Data are collected on the level of the child, his/her mother, father, and siblings. Most variables of interest will be assessed repeatedly over time; Fig. 1 depicts the measurement points of the study. Exposures before or during pregnancy are assessed retrospectively. Table 1 provides an overview of the domains covered by the study, including the most important exposures and outcomes as well as their respective assessment methods. In addition to general health outcomes of the child, a range of disease-specific outcomes is assessed (Table 2). Exposures and outcomes were selected to cover a broad range of health aspects in childhood based on results from previous studies such as Ulm Spatz [15, 16], KiGGS [17],
Table 2 Disease-specific outcomes currently investigated

\begin{tabular}{ll}
\hline & Diseases/syndromes \\
\hline Allergic diseases & Food allergy, asthma, hayfever, atopic dermatitis \\
Cardiac diseases & Long QT syndrome \\
$\begin{array}{l}\text { Dermatological } \\
\text { diseases }\end{array}$ & $\begin{array}{l}\text { Sequelae of sunburns, atopic dermatitis, erythema } \\
\text { toxicum neonatorum, skin lesions }\end{array}$ \\
$\begin{array}{l}\text { Gastroenterological } \\
\text { diseases }\end{array}$ & $\begin{array}{l}\text { Celiac disease, infantile colic, inflammatory bowel } \\
\text { disease }\end{array}$ \\
$\begin{array}{l}\text { Neurological } \\
\text { diseases }\end{array}$ & CNS infection, febrile seizure \\
Oral health & Tooth decay \\
Urological & Cryptorchidism, hypospadias, enuresis, urinary tract \\
disorders & infections, urolithiasis, hematuria, biliary atresia \\
\hline
\end{tabular}

ISAAC [18], and only recently identified influences on health such as exposure to digital media. Operationalization and assessment methods were selected to allow for joint analyses of data with two existing birth cohort studies from Germany (Ulm SPATZ $[15,16]$ and LIFE child in Leipzig [19]), and whenever available, validated questions and measurement instruments were used (see Table 2).

\section{Interview and questionnaires}

After inclusion in the study, a personal interview is conducted with the mother. All interviewers are medical doctoral students trained in study procedures and interview techniques according to a standardized training protocol. During follow-up, data are collected by self-report postal questionnaires, which include sections for

Table 1 Important domains, level of assessment, and assessment methods

\begin{tabular}{|c|c|c|c|c|c|}
\hline Domains & Child & Mother & Father & Sibling(s) & Assessment \\
\hline $\begin{array}{l}\text { Socio-demographic information: age, living circumstances, marital status, employment, } \\
\text { education, subjective social status (McArthur Scale [35]), health insurance }\end{array}$ & & $x$ & $x$ & & Interview; self-report \\
\hline $\begin{array}{l}\text { Pregnancy and birth: environmental exposure, medication, lifestyle behaviors, mode of } \\
\text { delivery, duration of pregnancy, complications }\end{array}$ & & $x$ & & & $\begin{array}{l}\text { Interview; self-report; } \\
\text { medical records }\end{array}$ \\
\hline Medical history: chronic conditions/diseases, hereditary diseases & & $x$ & $x$ & $x$ & $\begin{array}{l}\text { Interview; self-/proxy- } \\
\text { report }\end{array}$ \\
\hline $\begin{array}{l}\text { Health behaviors: (breast) feeding, nutrition, physical activity, sleep (CSHQ [36], PSQI [37]), } \\
\text { smoking, alcohol consumption, sun bathing and sun protection, teeth brushing, media } \\
\text { consumption }\end{array}$ & $(x)$ & $x$ & $x$ & & Self-/proxy-report \\
\hline $\begin{array}{l}\text { Home environment: exposure to allergens, measures to control allergens, smoking, } \\
\text { urbanity }\end{array}$ & $x$ & & & & Proxy-report \\
\hline $\begin{array}{l}\text { Psychosocial constructs: stress (EBI [38]), social support (F-SozU [39]), anxiety and } \\
\text { depression (PHQ-D [40]), health-related quality of life (SF-12 [41]), health literacy (HLS-EU [35]) }\end{array}$ & & $x$ & $x$ & & Self-report \\
\hline $\begin{array}{l}\text { Utilization of medical and non-medical services: health check-ups, vaccinations, ambulatory } \\
\text { and stationary health care, medication, early interventions, counseling }\end{array}$ & $x$ & $x$ & & & Self-/proxy-report \\
\hline $\begin{array}{l}\text { General health outcomes: health status, health-related quality of life, physical development, } \\
\text { mental development, obesity, accidents; specific diseases and syndromes }{ }^{a}\end{array}$ & $x$ & & & & $\begin{array}{l}\text { Clinical examination; } \\
\text { proxy-report }\end{array}$ \\
\hline
\end{tabular}

CSHQ Childhood Sleep Habits Questionnaire, EBI Parental Stress Index, F-SozU Social Support Questionnaire, HLS-EU European Health Literacy Survey, PSQI Pittsburgh Sleep Quality Index, SF-12 Short Form Health Survey

${ }^{a}$ For a comprehensive description of disease-specific outcomes considered in the KUNO-Kids birth cohort study, please see Table 2 
the study child, mother, father, and if applicable, for the sibling(s) of the study child.

\section{Clinical and anthropometric assessments}

Children participating in the study are assessed in the clinic after birth by a pediatrician according to a predefined standardized clinical procedure (based on an extended baby health check-up (called "U2-examination" in Germany) [20]) between 24 and $72 \mathrm{~h}$ after birth. The physical examination includes a whole body status, including the head, skin, chest, and abdominal organs, genitals, musculoskeletal and nervous systems as wells as the sensory organs. Anthropometric measurements of body length, weight, and head circumference are carried out. In addition, a standard 12-channel ECG is recorded and the oxygen saturation is assessed by pulse oximetry in order to screen for critical congenital heart defects.

\section{Sampling of biomaterials}

Biological samples of the child are collected at baseline. Because the study was specifically designed to collect biomaterials for omics approaches, a wide variety of material is collected according to predefined sampling procedures, taking into account (a) the limited amount of biosamples that can be collected from newborns and (b) the adherence to the principle of non-invasiveness in children. Therefore, neonatal blood was collected as cord blood, stool and urine were collected non-invasively by catching methods, and skin and buccal swabs were acquired. The time period for collecting samples such as cord blood is very restricted, and mothers' informed consent is gathered only after delivery. Therefore, cord blood samples are stored from all newborns until the parents have decided for or against participation in the study. If no informed consent to study participation and biosampling is present, stored samples are destroyed. Only samples of study participants are kept, stored, and worked up.

For all samples, digital bioprotocols for tracing sampling procedures, storage, and sample work up were developed. An overview of the sampling methods and subsequent analyses is provided in Table 3. The collection of samples and the pre-analytic procedures are performed according to standard operating procedures (SOPs) available from the authors upon request.

\section{Data management and protection}

Standardized interviews, questionnaires, and clinical assessment documents as well as biosample protocols were designed using QNOME - a novel software tool specifically developed for biomedical studies, which allows designing customized case report forms and questionnaires from a large range of available items and pre-existing questionnaires. Questionnaires are available as e-paper documents and/or online tools linking the data directly to an automatically generated multidimensional SQL database (available upon request by the authors). All study data are pseudonymized and stored on protected servers. Personal data (name, date of birth, address) are never linked to study data. Medical data and data from questionnaires and biosample ID tracking numbers, as well as results from bioanalyses, are stored separately and can only be linked using protected algorithms.

\section{Ethics and consent}

The study was approved by the Ethics Committee of the University of Regensburg (file number: 14-1010347). All participating parents provide written informed consent. The consent procedure which reflects all data sources and assessment methods is continuous over the course of the study so that at each follow-up, participants are asked if they will participate in the next follow-up. Furthermore, it provides the option of

Table 3 Biological sampling methods and analyses

\begin{tabular}{lll}
\hline Specimen & Purpose & Downstream applications \\
\hline Cord blood_EDTA & Genomic DNA isolation & Genotyping, sequencing, epigenetics (methylation) analyses \\
Cord blood_PAXgene & RNA and miRNA isolation & Transcriptomics \\
Cord blood_SSR (Clot Activator Tube) & Serum separation & Protein measurements: cytokines, allergens; metabolomics (NMR, LC-MS) \\
Cord blood_Li-Heparin & Plasma separation & Protein measurements: cytokines, allergens; metabolomics (NMR, LC-MS) \\
Stool & Bacterial DNA isolation & Microbiome analyses \\
Urine & & Metabolomics (NMR, LC-MS) \\
Skin swabs-cheek, elbow, forearm & Bacterial DNA isolation & Microbiome analyses \\
Buccal swabs & Genomic DNA isolation & Genotyping, sequencing, epigenetics (methylation) analyses \\
Buccal swabs & RNA and miRNA isolation & Transcriptomics \\
Hair & & Toxicology analyses \\
Gingival smears & Bacterial DNA isolation & Microbiome analyses \\
\hline
\end{tabular}


consenting to certain elements of the study while refusing consent to others (e.g., consenting to complete selfreport questionnaires, but declining the sampling of specific biomaterials). The participants have the right to withdraw from the study at any time.

\section{Results}

\section{Participation and response}

After 3 years of recruitment, 2515 infants and their families have joined the study. In addition to the general consent, which is a requirement for study participation, the vast majority of participating mothers also provided specific consent to additional study procedures and analyses: 99\% agreed to additional examinations of the infant (e.g., ECG, skin swabs), to the collection and analysis of biological samples, and to sharing data or biological samples with external researchers. Ninety-eight percent of participants consented to be re-contacted by the study team, and 97\% agreed to the analysis of genetic information.

Coverage of recruitment within the clinic St. Hedwig, participation rates, and reasons for non-participation were investigated in detail for a 2.5 -month time period between December 2017 and February 2018. Of all mothers giving birth in the clinic $(N=638)$ during this period, $73 \%(N=$ 465) were approached by study personnel and informed about the study. Reasons for failure to approach mothers were (a) acute and critical illness of mother or child, (b) discharge from hospital immediately after birth, or (c) organizational and health care-related issues such as mothers not available due to repeated absence from their hospital room, constant visitors, or necessary clinical examinations and therapies.

Of the mothers who were approached, $33 \%(N=154)$ agreed to participate in the study, $64 \%(N=291)$ could not be included, and $3 \%(N=20)$ met the exclusion criteria (minor; mother already participating in the study with other child). The most frequent reasons for nonparticipation were insufficient German language skills (31\%), mothers' perception that the study procedures are associated with too much effort (29\%), and lack of interest $(15 \%)$. Other reasons were personal or infant's ill health (5\%) or reluctance to delay being discharged from hospital due to the study (5\%).

Table 4 provides an overview of the response rates at various follow-up measurements during the child's first year of life. The largest study dropout occurs between baseline and the 4-week follow-up (36\%); at the 1-year follow-up, the response rate is about $50 \%$.

\section{Sample characteristics}

Socio-demographic characteristics of children, mothers, and fathers are presented in Table 5. Mothers' mean age is 33.9 years (standard deviation $(\mathrm{SD})=4.6$ ), and $56 \%$ of the mothers gave birth for the first time. The mean
Table 4 Response rates for different follow-up time points after 3 years of recruitment

\begin{tabular}{lll}
\hline Time point & Percentage & Number \\
\hline Baseline & $99^{\mathrm{a}}$ & 2492 (out of 2515) \\
4-week follow-up & $64^{\mathrm{b}}$ & 1618 (out of 2515) \\
6-month follow-up & $60^{\mathrm{b}}$ & 1260 (out of 2114) \\
1-year follow-up & $49^{\mathrm{b}}$ & 784 (out of 1588) \\
\hline
\end{tabular}

${ }^{\mathrm{a}} 100 \%(N=2515)$ refers to all participants who consented to participate in the study

${ }^{\mathrm{b}} 100 \%$ refer to those participants who reached the respective follow-up time point

educational level is high in both mothers and fathers, with more than half of participants having achieved a school leaving certificate that qualifies for university entrance. Nearly all fathers and more than half of mothers were employed full-time before the birth of their child. Eighty-five percent of mothers and $86 \%$ of fathers were born in Germany.

Study participants who returned the 1-year follow-up questionnaire differed from the baseline sample with regard to socio-demographic characteristics. Mothers with only one child, those with an older age or a higher education level, those with full-time employment before the birth of their child, and those without a migration background were more likely to have completed the 1-year follow-up questionnaire than their respective counterparts (Table 5).

\section{Discussion}

The KUNO-Kids study is a holistic "next generation" birth cohort which combines a broad phenotyping approach with a wide spectrum of omics technologies. The involvement of experts from various disciplines contributes to a study that covers a wide variety of topics with relevance for child health. This enables inter- and transdisciplinary research and is likely to generate novel findings. In order to advance the etiologic understanding of complex multi-factorial diseases, the KUNO-Kids study employs modern omics technologies. These provide the opportunity to better characterize both exposures and health outcomes and to consider the dynamics occurring in a life-course perspective.

One major advantage of such a holistic approach incorporating different research questions from different fields of medicine is that non-hypothesis driven but expensive omics technologies must be applied only once in the cohort but the omics data can then be used for many research questions, making the approach cost effective. However, it should be noted that the omics approach goes along with ethical (e.g., data privacy and protection) and scientific challenges-such as the integration of huge amounts of data from different levels or the consideration of omics data in risk assessment and prediction models- 
Table 5 Characteristics of included infants and their parents after 3 years of recruitment, separately for all study participants and for respondents at 1-year follow-up

\begin{tabular}{|c|c|c|c|c|}
\hline \multirow[b]{2}{*}{ Infants } & \multicolumn{2}{|c|}{$\begin{array}{l}\text { Participants at baseline } \\
(N=2492)\end{array}$} & \multicolumn{2}{|c|}{$\begin{array}{l}\text { Respondents at 1-year } \\
\text { follow-up }(N=784)^{\mathrm{b}}\end{array}$} \\
\hline & & & & \\
\hline Sex (female) $N(\%)$ & 2492 & $1223(49.1)$ & 784 & $393(50.1)$ \\
\hline Weight at birth (g) $M(S D)$ & 2479 & $3352(507)$ & 776 & $3343(503)$ \\
\hline Length at birth (cm) M (SD) & 2481 & $51(2.6)$ & 777 & $51.4(2.6)$ \\
\hline Duration of pregnancy (weeks) M (SD) & 2465 & $39.5(1.6)$ & 774 & $39.6(1.6)$ \\
\hline One or more older siblings $N(\%)$ & 2475 & $1091(44.0)$ & 775 & $304(39.2)$ \\
\hline \multicolumn{5}{|l|}{ Mothers } \\
\hline Maternal age (years) M (SD) & 2462 & $33.9(4.6)$ & 773 & $34.8(4.2)$ \\
\hline Maternal marital status & 2447 & & 766 & \\
\hline Married, living together with husband N (\%) & & $1919(78.4)$ & & $616(80.4)$ \\
\hline Unmarried, living together with partner (\%) & & $468(19.1)$ & & $136(17.8)$ \\
\hline Unmarried, without partner N (\%) & & $32(1.3)$ & & $8(1.0)$ \\
\hline Divorced N (\%) & & $26(1.1)$ & & $6(0.8)$ \\
\hline Widowed N (\%) & & $2(0.1)$ & & 0 \\
\hline Maternal education & 2437 & & 766 & \\
\hline School leaving certificate after less than 10 years of schooling $N(\%)$ & & $254(10.4)$ & & $54(7.0)$ \\
\hline School leaving certificate after 10 years of schooling $N(\%)$ & & $778(31.9)$ & & $272(35.5)$ \\
\hline University entrance level $N(\%)$ & & $1369(56.2)$ & & $498(65.0)$ \\
\hline Other school leaving certificate $N(\%)$ & & $16(0.7)$ & & $1(0.1)$ \\
\hline No school leaving certificate $N(\%)$ & & $20(0.8)$ & & $4(0.5)$ \\
\hline Maternal employment before birth & 2426 & & 760 & \\
\hline Full-time employed $N(\%)$ & & $1315(54.2)$ & & $466(61.3)$ \\
\hline Part-time employed $N(\%)$ & & $649(26.8)$ & & $193(25.4)$ \\
\hline Marginally/not regularly employed $N(\%)$ & & $82(3.4)$ & & $18(2.4)$ \\
\hline Maternal leave, housewife $N(\%)$ & & $243(10.0)$ & & $56(7.4)$ \\
\hline Pupil, student N (\%) & & $37(1.5)$ & & $16(2.1)$ \\
\hline Seeking for employment $N(\%)$ & & $21(0.9)$ & & $2(0.3)$ \\
\hline Other $N(\%)$ & & $79(3.3)$ & & $9(1.2)$ \\
\hline Born in Germany N (\%) & 2449 & $2075(84.7)$ & 768 & $690(89.8)$ \\
\hline Nationality & 2448 & & 768 & \\
\hline German N (\%) & & $2195(89.7)$ & & 719 (93.6) \\
\hline Other $N(\%)$ & & $197(8.1)$ & & $36(4.7)$ \\
\hline German and other $N(\%)$ & & $65(2.3)$ & & $13(1.7)$ \\
\hline \multicolumn{5}{|l|}{ Fathers } \\
\hline Paternal education & $1412^{\mathrm{a}}$ & & 643 & \\
\hline School leaving certificate after less than 10 years of schooling $N(\%)$ & & $234(16.6)$ & & $105(16.4)$ \\
\hline School leaving certificate after 10 years of schooling $N(\%)$ & & $304(21.5)$ & & $126(19.4)$ \\
\hline University entrance level $N(\%)$ & & $843(59.7)$ & & $401(62.4)$ \\
\hline Other school leaving certificate $N(\%)$ & & $15(1.1)$ & & $5(0.8)$ \\
\hline No school leaving certificate $N(\%)$ & & $16(1.1)$ & & $6(0.9)$ \\
\hline Paternal employment & $1486^{\mathrm{a}}$ & & 676 & \\
\hline Full-time employed $N(\%)$ & & $1367(92.0)$ & & $622(92.0)$ \\
\hline Part-time employed N (\%) & & $43(2.9)$ & & $24(3.6)$ \\
\hline
\end{tabular}


Table 5 Characteristics of included infants and their parents after 3 years of recruitment, separately for all study participants and for respondents at 1-year follow-up (Continued)

\begin{tabular}{|c|c|c|c|c|}
\hline & \multicolumn{2}{|c|}{$\begin{array}{l}\text { Participants at baseline } \\
(N=2492)\end{array}$} & \multicolumn{2}{|c|}{$\begin{array}{l}\text { Respondents at 1-year } \\
\text { follow-up }(N=784)^{\mathrm{b}}\end{array}$} \\
\hline Marginally/not regularly employed $N(\%)$ & & $8(0.5)$ & & $3(0.4)$ \\
\hline Paternal leave, househusband N (\%) & & $24(1.6)$ & & $10(1.5)$ \\
\hline Pupil, student N (\%) & & $22(1.5)$ & & $8(1.9)$ \\
\hline Seeking for employment $N(\%)$ & & $8(0.5)$ & & $4(0.6)$ \\
\hline Other $N(\%)$ & & $14(0.9)$ & & $6(0.9)$ \\
\hline Born in Germany N (\%) & 2427 & $2080(85.7)$ & 762 & $701(92.0)$ \\
\hline Nationality & $1457^{\mathrm{a}}$ & & 658 & \\
\hline German N (\%) & & $1360(93.3)$ & & $619(94.1)$ \\
\hline Other $N(\%)$ & & $74(5.1)$ & & $27(4.1)$ \\
\hline German and other $N(\%)$ & & $23(1.6)$ & & $12(1.8)$ \\
\hline
\end{tabular}

Notes: $M$ mean, $S D$ standard deviation, $N$ number of observations

a Low total $\mathrm{Ns}$ as this information was assessed not at baseline, but at 4-week follow-up

${ }^{b}$ Out of 1588 participants who already reached the 1 -year follow-up time point

and adequate procedures and statistical methods are only now developed [21, 22]. In addition, great emphasis needs to be placed on the pre-analytical sample recovery and work up to ensure a high quality of biomaterial entering these analyses. Thus, tracking of samples and standardized sample procedures are of uttermost importance for the integrity and quality of downstream omics data.

Overall, the socio-demographic characteristics of mothers and fathers in the KUNO-Kids study reflect a population from a prosperous region with good socio-economic living conditions for the majority of its inhabitants. Almost all study participants are employed or were employed before the birth of their child, which is in accordance with the very low rates of unemployment in Germany and especially in the south of Germany. However, the proportion of study participants with a high school leaving certificate is higher than expected, suggesting that such persons are more willing to participate in studies than their less educated counterparts [23]. Census data from the study region show that about $40 \%$ of women aged 20 to 39 years obtain a university entrance level (as compared to 56\% in our study) [24]. The same applies to the distribution of fathers' education in the study sample: $34 \%$ of men aged 20 to 39 years in the study area obtain a university entrance level [24] as compared to $60 \%$ of fathers in the study sample.

The recruitment of a socially diverse sample is a common challenge in population-based research. Migrants or socially disadvantaged people are often underrepresented in epidemiologic cohorts $[25,26]$. This is also the case in our study. Previous studies have identified barriers to participation in birth cohorts, such as limited language or literacy skills, burden imposed by excessive information or demanding study procedures, and lack of cultural sensitivity among study personnel $[27,28]$. The assessment of reasons for non-participation in the KUNO-
Kids study yielded similar findings. In approximately one third of the mothers approached, no informed consent could be achieved due to insufficient German language skills. Many different languages were spoken by the non-German speaking mothers, with no single language spoken by more than $5 \%$ of the women. This makes it difficult to design study procedures that would allow integrating non-German speaking families into the study as providing informed consent and understanding the dimension of the cohort study require unambiguous written and oral communication.

On the other hand, experience from other cohorts suggests that at least some barriers to study participation can be overcome by intensified recruitment efforts [27, 29, 30]. Considering the low participation rate in our study, we decided to modify the recruitment strategy in the next phase of the study. By informing and recruiting the mothers already during pregnancy, we acknowledge that some people wish to have more time for their decision about study participation and hope to motivate more families for long-term commitment to the study.

The longer the follow-up period, the more valuable are data derived from birth cohort studies. However, attrition bias caused by loss-to-follow-up is common in longitudinal studies leading to over- or underestimation of effects in case of differential loss-to-follow-up. This also applies to the KUNO-Kids study. The percentage of families who are lost between birth of the child and the 1-year follow-up measure is substantial, and the overrepresentation of well-educated families in the study sample increases over time. This increased selection bias due to attrition does not necessarily affect all exposure-outcomes associations, but it will where loss-to-follow-up is differential. As we saw that study retention is related to socio-economic status, associations between socio- 
economic status and outcomes [31] will likely be biased. Further, the validity of prevalence and incidence estimates may be compromised. Quantitative bias analysis will be undertaken to estimate the direction, magnitude, and uncertainty arising from systematic errors [32]. For the successful continuation of the study, it will be crucial to ensure that families which remained in the study for the child's first year of life continue to participate in the future. Measures which are currently implemented to further increase participants' long-term adherence to the study include the provision of online questionnaires for participants who prefer them over paper-based questionnaires, the partition of questionnaires into smaller portions, and the communication with study participants via regular e-mails with short communications about recent research findings and study news. Inviting participants for clinical follow-up visits in the study center could be a further means for maintaining contact between the study team and participants. These visits are envisioned, but not yet funded, and could be used for performing additional investigations and the validation of specific diagnoses.

Generally, people seem to have become leery with respect to the collection of biomaterial for scientific purposes and not all study participants consent to the collection and processing of biomaterial [33, 34]. In contrast, in the KUNO-Kids cohort, the majority of mothers agreed to the collection and examination of biological samples, including the analysis of genetic information. Against the background of the planned analyses, this high rate of consent is encouraging. It is likely that the hospital setting where both the recruitment of families and the non-invasive collection of biomaterial took place, as well as the above average education levels of the participating parents, the possibility of the continuous consent, and the option of withdrawal at any time contributed to the high acceptance rate. In the first phase of the KUNO-Kids study, the collection of biosamples is restricted to the index child. This limits the scope of research questions which can be addressed. However, many parental biosamples can be acquired at a later time point (e.g., for DNA analysis) when needed for specific research questions where biosamples in informative families can be collected using a case-control design to minimize costs of biomaterial sampling and biobanking. In the next phase of the study, when recruitment takes place in the last trimester of pregnancy, maternal biomaterial (blood, hair, saliva, urine, and microbiome samples) will be collected around delivery.

The KUNO-Kids birth cohort study is embedded in the routines of a maternity and children's hospital. Therefore, daily health care routines take precedence over study procedures and difficulties in the recruitment process arise from time to time due to incompatibilities between health care and research. However, the study's embedment in a hospital goes also along with some major advantages: It allows for comprehensive clinical assessments as well as the standardized collection and storage of biosamples-procedures whose implementation is often challenging in large population-based studies. This was achieved by a great effort in standardizing sample procedures going as far as providing ready-to-use sample sets for each biosample and designing digital biosample protocols with our QNOME system (www.qnome.de) which allowed tracking of samples and safely tagging information on sample recovery and storage to each individual sample by using barcodes wherever possible. Further, the study's integration in a health care setting can help limit the burden imposed on study participants by using existing routine health data (e.g., data from $\mathrm{U} 1$ and $\mathrm{U} 2$ health check-ups) and avoid additional examinations and assessments. However, this makes it necessary to standardize clinical procedures to study protocols and to perform documentation that exceeds standard clinical practice. Integrating digital devices into clinical practice and standardizing clinical procedures in KUNO-Kids was thus a prerequisite for the use of routine health data. For that purpose, the QNOME system was developed, which facilitates the implementation of digital documentation into clinical procedures. Routine health care data from clinical practice reflects the provision of actual health care under everyday conditions, and access to this data also enables health services research. Finally, the close collaboration between health care providers and researchers represents a starting point for using the birth cohort as a platform for developing and testing interventions in the setting of a cohort of well-informed and well-monitored study participants.

KUNO-Kids is designed as a single-center study. Although the study is based in a hospital where the majority of infants are born in a study region representative for many parts of Germany, generalizability to other populations such as metropolitan regions may be limited. To overcome this potential limitation, efforts were made from the beginning to harmonize assessment methods for exposures and outcomes to allow for a joint analysis with other birth cohorts in Germany.

\section{Conclusions}

Taken together, the data collected in the KUNO-Kids birth cohort study will provide extensive opportunities for investigating child health and its determinants in a holistic approach-in the study area and its adjacent regions as well as in a broader context via data pooling with other birth cohorts. After 3 years of recruitments, 2515 families have been included in the study. This suggests that the minimum aim of 5000 participants is 
feasible within the scheduled recruitment period of 5 to 10 years. However, like other long-term studies, the KUNO-Kids birth cohort study will need to put effort in continuing to successfully nurture the cohort and maintain participants' commitment to the study over time. The application of omics data to a wide range of diseases in the same sample bears the potential of identifying novel molecular and mechanistic patterns of health and disease well beyond currently established clinically defined concepts and borders allowing for novel concepts of understanding health and preventing disease development.

\section{Abbreviations}

CSHQ: Childhood Sleep Habits Questionnaire; EBI: Parental Stress Index; FSozU: Social Support Questionnaire; HLS-EU: European Health Literacy Survey; M: Mean; N: Number; PSQI: Pittsburgh Sleep Quality Index; SD: Standard deviation; SF-12: Short Form Health Survey

\section{Acknowledgements}

We thank all families who participate in the study and the medical students, nurses, midwives, physicians, laboratory personnel, and researchers who supported recruitment, examinations, and data assessment. Special thanks to Magdalena Scharl, Nico Bruns, Max Schieck, and Jon Genuneit for their contribution and support in setting up the study.

Further, we thank all members of the KUNO-Kids study group: Petra Arndt (ZNL Transfercenter of Neuroscience and Learning, University of UIm, UIm, Germany), Andrea Baessler (Department of Internal Medicine II, Regensburg University Medical Center, Regensburg, Germany), Mark Berneburg (Department of Dermatology, University Medical Centre Regensburg, Regensburg, Germany), Wolfgang Buchalla (Department of Conservative Dentistry and Periodontology, University Hospital Regensburg, University of Regensburg, Regensburg, Germany), Sara Fill Malfertheiner (Clinic of Obstetrics and Gynecology St. Hedwig, University of Regensburg, Regensburg, Germany), André Gessner (Department of Clinical Microbiology and Hygiene, University Hospital Regensburg, Regensburg, Germany), Iris Heid (Department of Genetic Epidemiology, University of Regensburg, Regensburg, Germany), Sebastian Kerzel (Department of Pediatric Pneumology and Allergy, University Children's Hospital Regensburg, St. Hedwig Campus, Regensburg, Germany), Michael Koller (Center for Clinical Studies, University Hospital Regensburg, Regensburg, Germany), Michael Leitzmann (Department of Epidemiology and Preventive Medicine, University of Regensburg, Regensburg, Germany), David Rothfuß (City of Regensburg, Coordinating Center for Early Interventions, Regensburg, Germany), Wolfgang Rösch (Department of Pediatric Urology, University Medical Center, Regensburg, Germany), Hugo Segerer (Children's Hospita St. Hedwig, University of Regensburg, Regensburg, Germany), Bernhard H.F. Weber (Institute of Human Genetics, University of Regensburg, Regensburg, Germany), and Stephan Weidinger (Department of Dermatology, Venereology and Allergy, University Hospital Schleswig-Holstein, Campus Kiel, Kiel, Germany).

\section{Funding}

The KUNO-Kids study is funded by research grants of the EU (HEALS: 603946) and the German Federal Ministry for Education and Research (SYSINFLAME: 01ZX1306E). Further financial support was provided by the University Children's Hospital of the University of Regensburg (KUNO-Clinics) and the clinic "St. Hedwig" (Hospital "Barmherzige Brüder Regensburg"). The funding bodies had neither influence on the design of the study, on the collection, analysis and interpretation of data, nor in the writing of the manuscript.

\section{Availability of data and materials}

The datasets used and/or analyzed for this paper are available from the corresponding author on reasonable request.

\section{Authors' contributions}

SB analyzed and interpreted the data and wrote the manuscript. AT drafted the sections of the manuscript dealing with all aspects of biosampling. JN helped with the sections on data management and protection. CW designed the assessment instruments and helped with the methods section. SG prepared the data on response and participation and helped with the methods section. BS-G conceptualized the design of the study and facilitated recruitment of participants in the clinic. CA contributed to the design of the study and helped interpret the data. MM contributed to the design of the study and facilitated recruitment of participants. MK designed the study and wrote the manuscript. All authors read and approved the final manuscript.

Ethics approval and consent to participate

The study has been approved by the Ethics Committee of the University of Regensburg (reference number: 14-101-0347). All participating parents provided written informed consent.

\section{Consent for publication}

Not applicable.

\section{Competing interests}

JN is head of the IT company ("Biteschmiede") which has developed the software tool for the KUNO-Kids study. All other authors declare that they have no competing interests.

\section{Publisher's Note}

Springer Nature remains neutral with regard to jurisdictional claims in published maps and institutional affiliations.

\section{Author details}

'University Children's Hospital Regensburg (KUNO-Clinics), University of Regensburg, Clinic St. Hedwig, Steinmetzstr. 1-3, 93049 Regensburg, Germany. ${ }^{2}$ Clinic of Obstetrics and Gynecology St. Hedwig, University of Regensburg, Regensburg, Germany. ${ }^{3}$ Medical Sociology, Institute of Epidemiology and Preventive Medicine, University of Regensburg, Regensburg, Germany.

Received: 27 September 2018 Accepted: 10 December 2018 Published online: 09 January 2019

\section{References}

1. Richmond RC, Al-Amin A, Smith GD et al (2014) Approaches for drawing causal inferences from epidemiological birth cohorts: a review. Early Hum Dev 90:769-780

2. Power C, Kuh D, Morton S (2013) From developmental origins of adult disease to life course research on adult disease and aging: insights from birth cohort studies. Annu Rev Public Health 34:7-28

3. Larsen PS, Kamper-Jorgensen M, Adamson A et al (2013) Pregnancy and birth cohort resources in europe: a large opportunity for aetiological child health research. Paediatr Perinat Epidemiol 27:393-414

4. Piler P, Kandrnal V, Blaha L (2017) Critical assessment of the research outcomes of European birth cohorts: linking environmental factors with non-communicable diseases. Public Health 145:136-145

5. Vrijheid M, Casas M, Bergstrom A et al (2012) European birth cohorts for environmental health research. Environ Health Perspect 120:29-37

6. Bunning M (2015) What happens after the 'Daddy Months'? Fathers' involvement in paid work, childcare, and housework after taking parental leave in Germany. Eur Sociol Rev 31:738-748

7. Oecd (2017). [Dare to share - Germany's experience promoting equal partnership in families] Doi.org/10.1787/9789264263420-4-de

8. Bmfsfj (2016). [Family and early education] www.bmfsfj.de/bmfsfj/servide/ publikationen/familie-und-fruehe-bildung/112460

9. Federal Statistical Office (Destatis) (2017). [Childlessness, births and families. Results from Mikrozensus 2016] https://www.destatis.de/DE/Publikationen/ Thematisch/Bevoelkerung/HaushalteMikrozensus/ GeburtentrendsTabellenband5122203169014.pdf?_blob=publicationFile

10. Coyne SM, Radesky J, Collier KM et al (2017) Parenting and digital media. Pediatrics 140:S112-S116

11. Medienpädagogischer Forschungsverbund Südwest (Mpfs) (2016). [Family Interaction, Media (FIM study)] www.mpfs.de/studien/fim-studie/2016

12. Heals Consortium Health and Environment-wide Associations based on Large population Surveys. The Largest Research Project In Europe On Environment And Health www.heals-eu.eu 
13. Regierung Der Oberpfalz (2018). Oberpfalz in Zahlen [Upper Palatinate in Figures] www.regierung.oberpfalz.bayern.de/landesplanung/statistik/oiz_ kompakt.pdf

14. City of Regensburg DOS (2017). Statistisches Jahrbuch 2017 [Statistical Yearbook 2017] http://www.statistik.regensburg.de/

15. Braig S, Weiss JM, Stalder T et al (2017) Maternal prenatal stress and child atopic dermatitis up to age 2 years: the UIm SPATZ health study. Pediatr Allergy Immunol 28:144-151

16. Logan C, Zittel T, Striebel S et al (2016) Changing societal and lifestyle factors and breastfeeding patterns over time. Pediatrics 137

17. Kurth BM, Kamtsiuris $\mathrm{P}$, Holling $\mathrm{H}$ et al (2008) The challenge of comprehensively mapping children's health in a nation-wide health survey: design of the German KiGGS-study. BMC Public Health 8:196

18. Genuneit J, Cantelmo JL, Weinmayr G et al (2009) A multi-Centre study of candidate genes for wheeze and allergy: the international study of asthma and allergies in childhood phase 2. Clin Exp Allergy 39:1875-1888

19. Quante M, Hesse M, Dohnert M et al (2012) The LIFE child study: a life course approach to disease and health. BMC Public Health 12:1021

20. Gemeinsamer Bundesausschuss (G-Ba) [Federal Joint Committee (G-Ba)] Richtlinie des Gemeinsamen Bundesausschusses über die Früherkennung von Krankheiten bei Kindern (Kinder-Richtlinie) [Guideline of the Federal Joint Committee on early diagonsis and screening of diseases in children] https://www.g-ba.de/downloads/ 62-492-1537/RL_Kinder_2017-10-19_iK-2018-03-16.pdf

21. Coughlin SS (2014) Toward a road map for global -omics: a primer on -omic technologies. Am J Epidemiol 180:1188-1195

22. Lopez De Maturana E, Pineda S, Brand A et al (2016) Toward the integration of omics data in epidemiological studies: still a "long and winding road". Genet Epidemiol 40:558-569

23. Galea S, Tracy M (2007) Participation rates in epidemiologic studies. Ann Epidemiol 17:643-653

24. Statistisches Bundesamt [German Federal Statistical Office] Microcensus 2011, city and region Regensburg. Extrapolation for 2014

25. Grosser A, Razum O, Vrijkotte TG et al (2016) Inclusion of migrants and ethnic minorities in European birth cohort studies-a scoping review. Eur J Pub Health 26:984-991

26. Winkler $\mathrm{V}$, Leitzmann $\mathrm{M}$, Obi $\mathrm{N}$ et al (2014) Response in individuals with and without foreign background and application to the National Cohort in Germany: which factors have an effect? Int J Public Health 59:555-563

27. Arora A, Manohar N, Bedros D et al (2018) Lessons learnt in recruiting disadvantaged families to a birth cohort study. BMC Nurs 17:7

28. Brumatti LV, Montico M, Russian S et al (2013) Analysis of motivations that lead women to participate (or not) in a newborn cohort study. BMC Pediatr 13:53

29. Golding J, Birmingham K (2009) Enrolment and response rates in a longitudinal birth cohort. Paediatr Perinat Epidemiol 23:73-85

30. Spallek J, Grosser A, Holler-Holtrichter C et al (2017) Early childhood health in Bielefeld, Germany (BaBi study): study protocol of a socialepidemiological birth cohort. BMJ Open 7:e018398

31. Howe LD, Tilling K, Galobardes B et al (2013) Loss to follow-up in cohort studies: bias in estimates of socioeconomic inequalities. Epidemiology 24:1-9

32. Lash TL, Fox MP, Maclehose RF et al (2014) Good practices for quantitative bias analysis. Int J Epidemiol 43:1969-1985

33. Ebner A, Thyrian JR, Lange A et al (2010) Survey of neonates in Pomerania (SNiP): a population-based birth study--objectives, design and population coverage. Paediatr Perinat Epidemiol 24:190-199

34. Ernst SA, Gunther K, Frambach T et al (2015) Prenatal recruitment of participants for a birth cohort study including cord blood collection: results of a feasibility study in Bremen, Germany. Ger Med Sci 13:Doc04

35. Sørensen K, Van Den Broucke S, Pelikan JM et al (2013) Measuring health literacy in populations: illuminating the design and development process of the European health literacy survey questionnaire (HLS-EU-Q). BMC Public Health 13:948

36. Owens JA, Spirito A, Mcguinn M (2000) The Children's sleep habits questionnaire (CSHQ): psychometric properties of a survey instrument for school-aged children. Sleep 23:1043-1051
37. Buysse DJ, Reynolds CF 3rd, Monk TH et al (1989) The Pittsburgh sleep quality index: a new instrument for psychiatric practice and research. Psychiatry Res 28:193-213

38. Tröster H (2010) Eltern-Belastungs-Inventar [parenting stress index] (manual). Hogrefe, Göttingen

39. Fydrich T, Sommer G, Brähler E (2007) Social support questionnaire (F-SozU). Manual. Hogrefe, Göttingen

40. Löwe B, Spitzer RL, Zipfel S, Herzog W (2002) PHQ-D Health Questionnaire for Patients. Manual. Pfizer $\mathrm{GmbH}$, Karlsruhe

41. Ware J Jr, Kosinski M, Keller SD (1996) A 12-item short-form health survey: construction of scales and preliminary tests of reliability and validity. Med Care 34:220-233

\section{Submit your manuscript to a SpringerOpen ${ }^{\circ}$ journal and benefit from:}

- Convenient online submission

- Rigorous peer review

- Open access: articles freely available online

- High visibility within the field

- Retaining the copyright to your article

Submit your next manuscript at $>$ springeropen.com 